\title{
New equalities and inequalities of $K$-g-frames in subspaces
}

\author{
Xiang-Chun Xiao ${ }^{\mathrm{a}, *}$, Guo-Rong Zhou ${ }^{\mathrm{a}}$, Yu-Can Zhu ${ }^{\mathrm{b}}$ \\ ${ }^{a}$ Department of Mathematics, Xiamen University of Technology, Xiamen 361024, China \\ b Department of Mathematics and Computer Science, Fuzhou University, Fuzhou 350116, China
}

*Corresponding author, e-mail: xxc570@163.com

Received 4 Sep 2018

Accepted 27 Aug 2019

\begin{abstract}
In this paper, we present new types of equalities and inequalities for $K$-g-frames in Hilbert spaces. Our equalities and inequalities for $K$-g-frames are different from previous ones for $g$-frames or fusion frames, owing to the fact that the bounded linear operator $K$ and a parameter $\lambda$ are involved, and thus allowing several known results to be derivable from our results by proper choices of $K$ and $\lambda$.
\end{abstract}

KEYWORDS: $g$-Bessel sequence, equality, inequality, $K$-dual

MSC2010: 42C15

\section{INTRODUCTION}

Sun ${ }^{1}$ introduced a frame, later named the $g$-frame, in order to deal with existing frames, such as frames $^{2}$, pseudo-frames ${ }^{3}$, and fusion frames ${ }^{4,5}$ as a united single object. Nowadays, $g$-frames ${ }^{6-8}$ are widely studied by many authors. We refer the readers to Refs. 9-11 for more information on $g$ frames.

In this paper, we obtain some new equalities and inequalities for $K$ - $g$-frames in Hilbert spaces. The $K$ $\mathrm{g}$-frame was first introduced by Xiao et al in Ref. 12 to generalize $g$-frames and $K$-frames ${ }^{13}$. It should be noted that some properties of $K$-g-frames are quite different from those of $g$-frames and $K$-frames. For more details on $\mathrm{K}$-g-frames, the readers can check Ref. 10.

Although there are a lot of literatures on equalities and inequalities for frames ${ }^{14,15}$, fusion frames ${ }^{16}$ and $g$-frames ${ }^{17,18}$, we present some new relations involving a parameter $\lambda$ for $K$ - $g$-frames, inspired by the work of Poria ${ }^{19}$. Several known results can be obtained by assigning specific values to $\lambda$ (see Remark 1, Remark 2, and Remark 3). Note that the equalities and inequalities for $K$-g-frames obtained in this paper contain the bounded linear operator $K$ and a parameter $\lambda$, and so are different from previous ones obtained for $g$-frames or fusion frames (e.g., Refs. 14, 17, 19).

Throughout this paper, we adopt the following notation:s $\mathscr{U}$ and $\mathscr{V}$ are Hilbert spaces, with inner product $\langle\cdot, \cdot\rangle$, and norm $\|\cdot\|$; the identity operator of $\mathscr{U}$ is denoted by $I_{\mathscr{U}} ; L(\mathscr{U}, \mathscr{V})$ denotes the collection of all linear bounded operators from $\mathscr{U}$ to $\mathscr{V}$, if $\mathscr{U}=\mathscr{V}$, then $L(\mathscr{U}, \mathscr{V})$ is shortened to $L(\mathscr{U})$. If $K \in L(\mathscr{H})$, then the range and the kernel of $K$ are denoted by $R(K)$ and $N(K)$, respectively.

\section{PRELIMINARIES OF $K$-G-FRAMES}

In this section we mainly recall some preliminaries of $K$-g-frames in Hilbert spaces.

Definition 1 [Ref. 1] A sequence $\left\{\Lambda_{j} \in L\left(\mathscr{U}, \mathscr{V}_{j}\right)\right.$ : $j \in J\}$ is called a $g$-frame for $\mathscr{U}$ with respect to (w.r.t.) $\left\{\mathscr{V}_{j}: j \in J\right\}$, if there exist two positive constants $A$ and $B$ such that

$$
A\|f\|^{2} \leqslant \sum_{j \in J}\left\|\Lambda_{j} f\right\|^{2} \leqslant B\|f\|^{2}, \quad \forall f \in \mathscr{U} .
$$

$A$ and $B$ are called the lower and the upper frame bounds of $\left\{\Lambda_{j}: j \in J\right\}$. We call $\left\{\Lambda_{j}: j \in J\right\}$ the $g$-Bessel sequence if only the right-hand inequality of (1) holds. If $\sum_{j \in J}\left\|\Lambda_{j} f\right\|^{2}=A\|f\|^{2}, A>0$, then $\left\{\Lambda_{j}: j \in J\right\}$ is called a tight $g$-frame with frame bound $A$.

Definition 2 [Ref. 12] A sequence $\left\{\Lambda_{j} \in L\left(\mathscr{U}, \mathscr{V}_{j}\right)\right.$ : $j \in J\}$ is called a $K$-g-frame for $\mathscr{U}$ w.r.t. $\left\{\mathscr{V}_{j}: j \in J\right\}$, if there exist $A, B>0$ such that

$$
A\left\|K^{*} f\right\|^{2} \leqslant \sum_{j \in J}\left\|\Lambda_{j} f\right\|^{2} \leqslant B\|f\|^{2}, \quad \forall f \in \mathscr{U} .
$$


We call $A$ and $B$ the lower and the upper frame bounds for the $K$-g-frame $\left\{\Lambda_{j}: j \in J\right\}$, respectively.

For a $g$-Bessel sequence $\left\{\Lambda_{j}: j \in J\right\}$ in $\mathscr{U}$ w.r.t. $\left\{\mathscr{V}_{j}: j \in J\right\}$, if there exists $A>0$ such that

$$
\sum_{j \in J}\left\|\Lambda_{j} f\right\|^{2}=A\left\|K^{*} f\right\|^{2}, \quad \forall f \in \mathscr{U},
$$

then we call $\left\{\Lambda_{j}: j \in J\right\}$ a tight $K$-g-frame with frame bound $A$.

Assume that $\left\{\Lambda_{j} \in L\left(\mathscr{U}, \mathscr{V}_{j}\right): j \in J\right\}$ is a $g$-Bessel sequence in $\mathscr{U}$. Then the frame operator of $\left\{\Lambda_{j}: j \in\right.$ $J\}$ is defined as follows.

$$
S: \mathscr{U} \rightarrow \mathscr{U}, \quad S f=\sum_{j \in J} \Lambda_{j}^{*} \Lambda_{j} f, \quad \forall f \in \mathscr{U} .
$$

Let $\left\{\Lambda_{j} \in L\left(\mathscr{U}, \mathscr{V}_{j}\right): j \in J\right\}$ be a $K$-g-frame for $\mathscr{U}$. According to Ref. 12 , there exists a $g$-Bessel sequence $\left\{\Gamma_{j} \in L\left(\mathscr{U}, \mathscr{V}_{j}\right): j \in J\right\}$ in $\mathscr{U}$ such that

$$
K f=\sum_{j \in J} \Lambda_{j}^{*} \Gamma_{j} f, \quad \forall f \in \mathscr{U} .
$$

$\left\{\Gamma_{j}\right\}_{j \in J}$ is called a $K$-dual of $\left\{\Lambda_{j}\right\}_{j \in J}$. Note that the roles of $\left\{\Gamma_{j}\right\}_{j \in J}$ and $\left\{\Lambda_{j}\right\}_{j \in J}$ are not interchangeable in general ${ }^{12}$. By (4) and the $K$-dual definition (5), we can obtain the following interesting result.

Proposition 1 For any g-Bessel sequence $\left\{\Lambda_{j}\right\}_{j \in J}$ with frame operator $S,\left\{\Lambda_{j}\right\}_{j \in J}$ is an $S$-dual of $\left\{\Lambda_{j}\right\}_{j \in J}$.

Proof: It follows from (4) and (5).

We also need the following lemmas.

Lemma 1 (Ref. 2) Suppose that $\mathscr{H}_{1}$ and $\mathscr{H}_{2}$ are two Hilbert spaces, and $Q \in L\left(\mathscr{H}_{1}, \mathscr{H}_{2}\right)$ has closed range. Then, there exists a unique bounded operator $Q^{+}: \mathscr{H}_{2} \rightarrow \mathscr{H}_{1}$, called the pseudo-inverse operator of $Q$, satisfying

$$
\begin{array}{cl}
N\left(Q^{+}\right)=R(Q)^{\perp}, & R\left(Q^{+}\right)=N(Q)^{\perp}, \\
Q Q^{+}=P_{R(Q)}, & Q^{+} Q=P_{R\left(Q^{+}\right)} .
\end{array}
$$

Lemma 2 (Ref. 19) Suppose that $P, Q \in L(\mathscr{U})$ satisfy $P+Q=I_{\mathscr{U}}$. Then, for any $\lambda \in[0,1]$, we have

$$
\begin{aligned}
P^{*} P+\lambda\left(Q^{*}+Q\right) & =Q^{*} Q+(1-\lambda)\left(P^{*}+P\right)+(2 \lambda-1) I_{\mathscr{U}} \\
& \geqslant\left(1-(\lambda-1)^{2}\right) I_{\mathscr{U}} .
\end{aligned}
$$

Lemma 3 Suppose that $P, Q \in L(\mathscr{U})$ satisfy $P+Q=$ $I_{\mathscr{U}}$. Then for any $\lambda$ we have

$$
P^{*} P+\lambda P+Q^{*}=Q^{*} Q-(1+\lambda) Q+(\lambda+1) I_{\mathscr{U}} .
$$

Proof: For any $\lambda$, we have

$$
\begin{aligned}
P^{*} P+\lambda P+Q^{*} & =\left(I_{\mathscr{U}}-Q\right)^{*}\left(I_{\mathscr{U}}-Q\right)+\lambda\left(I_{\mathscr{U}}-Q\right)+Q^{*} \\
& =Q^{*} Q-(1+\lambda) Q+(\lambda+1) I_{\mathscr{U}} .
\end{aligned}
$$

Lemma 4 Suppose that $P, Q \in L(\mathscr{U})$ and $P+Q=M$. Then we have

$$
P^{*} P+M^{*} M=Q^{*} Q+M^{*} P+P^{*} M .
$$

Proof: The result follows from

$$
\begin{aligned}
Q^{*} Q+M^{*} P+P^{*} M & =(M-P)^{*}(M-P)+M^{*} P+P^{*} M \\
& =P^{*} P+M^{*} M .
\end{aligned}
$$

\section{EQUALITIES AND INEQUALITIES OF} $K$-G-FRAMES

For a pair of $K$-dual $\left\{\Lambda_{j}\right\}_{j \in J}$ and $\left\{\Gamma_{j}\right\}_{j \in J}$, if $R(K)$ is closed, we can obtain the following equalities and inequalities with $K^{+}$and a parameter $\lambda$.

Theorem 1 Let $\left\{\Lambda_{j}\right\}_{j \in J}$ be a K-g-frame for $\mathscr{U}$ w.r.t $\left\{\mathscr{V}_{j}: j \in J\right\}$. Let $\left\{\Gamma_{j}\right\}_{j \in J}$ be a $g$-Bessel sequence in $\mathscr{U}$ w.r.t. $\left\{\mathscr{V}_{j}: j \in J\right\}$ and be a $K$-dual of $\left\{\Lambda_{j}\right\}_{j \in J}$. If $R(K)$ is closed, then we obtain:

(i) For any $f \in R(K),\left\{a_{j}\right\}_{j \in J} \in l^{\infty}(J)$, and $\lambda \in$ $[0,1]$,

$$
\begin{gathered}
\left\|\sum_{j \in J} a_{j} \Lambda_{j}^{*} \Gamma_{j} K^{+} f\right\|^{2}+2 \lambda \operatorname{Re} \sum_{j \in J}\left(1-a_{j}\right)\left\langle\Gamma_{j} K^{+} f, \Lambda_{j} f\right\rangle \\
=\left\|\sum_{j \in J}\left(1-a_{j}\right) \Lambda_{j}^{*} \Gamma_{j} K^{+} f\right\|^{2} \\
+2(1-\lambda) \operatorname{Re} \sum_{j \in J} a_{j}\left\langle\Gamma_{j} K^{+} f, \Lambda_{j} f\right\rangle+(2 \lambda-1)\|f\|^{2} \\
\geqslant\left(1-(\lambda-1)^{2}\right)\|f\|^{2} .
\end{gathered}
$$

(ii) For any $f \in R(K),\left\{a_{j}\right\}_{j \in J} \in l^{\infty}(J), \lambda \in \mathbb{R}$, and $\lambda \in[-1,3]$,

$$
\begin{aligned}
&\left\|\sum_{j \in J} a_{j} \Lambda_{j}^{*} \Gamma_{j} K^{+} f\right\|^{2}+\lambda \sum_{j \in J} a_{j}\left\langle\Gamma_{j} K^{+} f, \Lambda_{j} f\right\rangle \\
&+\sum_{j \in J}\left(1-\bar{a}_{j}\right)\left\langle\Lambda_{j} f, \Gamma_{j} K^{+} f\right\rangle \\
&=\left\|\sum_{j \in J}\left(1-a_{j}\right) \Lambda_{j}^{*} \Gamma_{j} K^{+} f\right\|^{2} \\
&-(1+\lambda) \sum_{j \in J}\left(1-a_{j}\right)\left\langle\Gamma_{j} K^{+} f, \Lambda_{j} f\right\rangle \\
&+(\lambda+1)\|f\|^{2} .
\end{aligned}
$$


Furthermore, we obtain

$$
\begin{aligned}
&\left\|\sum_{j \in J} a_{j} \Lambda_{j}^{*} \Gamma_{j} K^{+} f\right\|^{2}+\lambda \operatorname{Re} \sum_{j \in J} a_{j}\left\langle\Gamma_{j} K^{+} f, \Lambda_{j} f\right\rangle \\
&+\operatorname{Re} \sum_{j \in J}\left(1-\bar{a}_{j}\right)\left\langle\Lambda_{j} f, \Gamma_{j} K^{+} f\right\rangle \\
& \geqslant\left(1-\frac{1}{4}(\lambda-1)^{2}\right)\|f\|^{2} \quad \text { or } \\
&\left(\frac{3}{4}-\frac{1}{4} \lambda\right)\left\|\sum_{j \in J}\left(1-a_{j}\right) \Lambda_{j}^{*} \Gamma_{j} K^{+} f\right\|^{2} .
\end{aligned}
$$

Proof: For any $f \in \mathscr{U}$ and $\left\{a_{j}\right\}_{j \in J} \in l^{\infty}(J)$, define $L_{1}, L_{2}: \mathscr{U} \rightarrow \mathscr{U}$ as follows.

$$
L_{1} f=\sum_{j \in J} a_{j} \Lambda_{j}^{*} \Gamma_{j} f, \quad L_{2} f=\sum_{j \in J}\left(1-a_{j}\right) \Lambda_{j}^{*} \Gamma_{j} f .
$$

We now show that $L_{1}$ and $L_{2}$ are well defined. For any $f \in \mathscr{U}$ and any finite subset $I \subset J$, we have

$$
\begin{aligned}
& \left\|\sum_{j \in I} a_{j} \Lambda_{j}^{*} \Gamma_{j} f\right\|=\sup _{g \in \mathscr{U},\|g\|=1}\left|\left\langle\sum_{j \in I} a_{j} \Lambda_{j}^{*} \Gamma_{j} f, g\right\rangle\right| \\
& =\sup _{g \in \mathscr{U},\|g\|=1}\left|\sum_{j \in I} a_{j}\left\langle\Gamma_{j} f, \Lambda_{j} g\right\rangle\right| \\
& \leqslant \sup _{g \in \mathscr{U},\|g\|=1}\|a\|_{\infty} \sum_{j \in I}\left\|\Gamma_{j} f\right\|\left\|\Lambda_{j} g\right\| \\
& \leqslant\|a\|_{\infty} \sup _{g \in \mathscr{U},\|g\|=1}\left(\sum_{j \in I}\left\|\Gamma_{j} f\right\|^{2}\right)^{1 / 2}\left(\sum_{j \in I}\left\|\Lambda_{j} g\right\|^{2}\right)^{1 / 2} \\
& \leqslant\|a\|_{\infty} \sup _{g \in \mathscr{U},\|g\|=1} \sqrt{B}\|g\|\left(\sum_{j \in I}\left\|\Gamma_{j} f\right\|^{2}\right)^{1 / 2} \\
& \quad=\sqrt{B}\|a\|_{\infty}\left(\sum_{j \in I}\left\|\Gamma_{j} f\right\|^{2}\right)^{1 / 2}
\end{aligned}
$$

where $B$ is the Bessel bound of $\left\{\Lambda_{j}\right\}_{j \in J}$. As $\left\{\Gamma_{j}\right\}_{j \in J}$ is a $g$-Bessel sequence in $\mathscr{U}$ and $I$ is an arbitrary finite subset of $J, L_{1}$ is well defined on $\mathscr{U}$. Similarly we can also show that $L_{2}$ is well defined on $\mathscr{U}$.

As $\left\{\Gamma_{j}\right\}_{j \in J}$ is a $K$-dual of $\left\{\Lambda_{j}\right\}_{j \in J}$, we have

$$
L_{1}+L_{2}=K
$$

Also, as $R(K)$ is closed, from Lemma 1 and (11), we have

$$
L_{1} K^{+}+L_{2} K^{+}=K K^{+}=P_{R(K)} .
$$

It follows that

$$
\left.L_{1} K^{+}\right|_{R(K)}+\left.L_{2} K^{+}\right|_{R(K)}=I_{R(K)} .
$$

For (i), from Lemma 2 and (13), we obtain

$$
\begin{aligned}
\left(\left.L_{1} K^{+}\right|_{R(K)}\right)^{*}\left(\left.L_{1} K^{+}\right|_{R(K)}\right) & \\
& \quad+\lambda\left[\left(\left.L_{2} K^{+}\right|_{R(K)}\right)^{*}+\left.L_{2} K^{+}\right|_{R(K)}\right] \\
= & \left(\left.L_{2} K^{+}\right|_{R(K)}\right)^{*}\left(\left.L_{2} K^{+}\right|_{R(K)}\right) \\
& +(1-\lambda)\left[\left(\left.L_{1} K^{+}\right|_{R(K)}\right)^{*}+\left.L_{1} K^{+}\right|_{R(K)}\right] \\
& +(2 \lambda-1) I_{R(K)} \\
\geqslant & \left(1-(\lambda-1)^{2}\right) I_{R(K)} .
\end{aligned}
$$

And for any $f \in R(K)$, we have

$$
\begin{aligned}
\left\langle\left(\left.L_{1} K^{+}\right|_{R(K)}\right)^{*}\left(\left.L_{1} K^{+}\right|_{R(K)}\right) f, f\right\rangle & \\
& +\lambda\left\langle\left(\left(\left.L_{2} K^{+}\right|_{R(K)}\right)^{*}+\left.L_{2} K^{+}\right|_{R(K)}\right) f, f\right\rangle \\
= & \left\|L_{1} K^{+} f\right\|^{2}+\lambda\left\langle f, L_{2} K^{+} f\right\rangle+\lambda\left\langle L_{2} K^{+} f, f\right\rangle \\
= & \left\|L_{1} K^{+} f\right\|^{2}+2 \lambda \operatorname{Re}\left\langle L_{2} K^{+} f, f\right\rangle \\
= & \left\|\sum_{j \in J} a_{j} \Lambda_{j}^{*} \Gamma_{j} K^{+} f\right\|^{2} \\
& \quad+2 \lambda \operatorname{Re} \sum_{j \in J}\left(1-a_{j}\right)\left\langle\Gamma_{j} K^{+} f, \Lambda_{j} f\right\rangle,
\end{aligned}
$$

and

$$
\begin{aligned}
& \left\langle\left(\left.L_{2} K^{+}\right|_{R(K)}\right)^{*}\left(\left.L_{2} K^{+}\right|_{R(K)}\right) f, f\right\rangle \\
& +(1-\lambda)\left\langle\left(\left(\left.L_{1} K^{+}\right|_{R(K)}\right)^{*}+\left.L_{1} K^{+}\right|_{R(K)}\right) f, f\right\rangle \\
& +(2 \lambda-1)\left\langle I_{R(K)} f, f\right\rangle \\
& =\left\|L_{2} K^{+} f\right\|^{2}+2(1-\lambda) \operatorname{Re}\left\langle L_{1} K^{+} f, f\right\rangle+(2 \lambda-1)\|f\|^{2} \\
& =\left\|\sum_{j \in J}\left(1-a_{j}\right) \Lambda_{j}^{*} \Gamma_{j} K^{+} f\right\|^{2} \\
& +2(1-\lambda) \operatorname{Re} \sum_{j \in J} a_{j}\left\langle\Gamma_{j} K^{+} f, \Lambda_{j} f\right\rangle+(2 \lambda-1)\|f\|^{2} .
\end{aligned}
$$

Combining with (14) and (15), we know that (9) holds. For (ii), from (13) and Lemma 3, we have for any $\lambda$,

$$
\begin{gathered}
\left(\left.L_{1} K^{+}\right|_{R(K)}\right)^{*}\left(\left.L_{1} K^{+}\right|_{R(K)}\right)+\lambda\left(\left.L_{1} K^{+}\right|_{R(K)}\right)+\left(\left.L_{2} K^{+}\right|_{R(K)}\right)^{*} \\
=\left(\left.L_{2} K^{+}\right|_{R(K)}\right){ }^{*}\left(\left.L_{2} K^{+}\right|_{R(K)}\right) \\
\quad-(1+\lambda)\left(\left.L_{2} K^{+}\right|_{R(K)}\right)+(\lambda+1) I_{R(K)} .
\end{gathered}
$$

Hence for any $f \in R(K)$, we have

$$
\begin{aligned}
& \left\|L_{1} K^{+} f\right\|^{2}+\lambda\left\langle\left(L_{1} K^{+}\right) f, f\right\rangle+\left\langle\left(L_{2} K^{+}\right)^{*} f, f\right\rangle \\
& \quad=\left\|\left(L_{2} K^{+}\right) f\right\|^{2}-(1+\lambda)\left\langle\left(L_{2} K^{+}\right) f, f\right\rangle+(\lambda+1)\|f\|^{2} .
\end{aligned}
$$


It follows that (10) holds. Furthermore, we obtain

$$
\begin{gathered}
\left\|\sum_{j \in J} a_{j} \Lambda_{j}^{*} \Gamma_{j} K^{+} f\right\|^{2}+\lambda \operatorname{Re} \sum_{j \in J} a_{j}\left\langle\Gamma_{j} K^{+} f, \Lambda_{j} f\right\rangle \\
\quad+\operatorname{Re} \sum_{j \in J}\left(1-\bar{a}_{j}\right)\left\langle\Lambda_{j} f, \Gamma_{j} K^{+} f\right\rangle \\
=\left\|L_{1} K^{+} f\right\|^{2}+\lambda \operatorname{Re}\left\langle\left(L_{1} K^{+}\right) f, f\right\rangle+\operatorname{Re}\left\langle\left(L_{2} K^{+}\right)^{*} f, f\right\rangle \\
=\left\|\left(L_{2} K^{+}\right) f\right\|^{2}-(1+\lambda) \operatorname{Re}\left\langle\left(L_{2} K^{+}\right) f, f\right\rangle+(\lambda+1)\|f\|^{2} \\
=\left\|\left(L_{2} K^{+}\right) f-\frac{1+\lambda}{2} f\right\|^{2}+\left(\lambda+1-\frac{(1+\lambda)^{2}}{4}\right)\|f\|^{2} \\
\geqslant\left(1-\frac{1}{4}(\lambda-1)^{2}\right)\|f\|^{2} .
\end{gathered}
$$

From (17) we also obtain

$$
\begin{gathered}
\left\|\sum_{j \in J} a_{j} \Lambda_{j}^{*} \Gamma_{j} K^{+} f\right\|^{2}+\lambda \operatorname{Re} \sum_{j \in J} a_{j}\left\langle\Gamma_{j} K^{+} f, \Lambda_{j} f\right\rangle \\
\quad+\operatorname{Re} \sum_{j \in J}\left(1-\bar{a}_{j}\right)\left\langle\Lambda_{j} f, \Gamma_{j} K^{+} f\right\rangle \\
=\left\|\left(L_{2} K^{+}\right) f\right\|^{2}-(1+\lambda) \operatorname{Re}\left\langle\left(L_{2} K^{+}\right) f, f\right\rangle+(\lambda+1)\|f\|^{2} \\
=(1+\lambda)\left(\|f\|^{2}-\operatorname{Re}\left\langle\left(L_{2} K^{+}\right) f, f\right\rangle\right)+\left\|\left(L_{2} K^{+}\right) f\right\|^{2} \\
=(1+\lambda)\left(\left\|f-\frac{1}{2} L_{2} K^{+} f\right\|^{2}-\frac{1}{4}\left\|\left(L_{2} K^{+}\right) f\right\|^{2}\right) \\
\quad+\left\|\left(L_{2} K^{+}\right) f\right\|^{2} \\
=(1+\lambda)\left\|f-\frac{1}{2} L_{2} K^{+} f\right\|^{2}+\left(\frac{3}{4}-\frac{1}{4} \lambda\right)\left\|\left(L_{2} K^{+}\right) f\right\|^{2} \\
\geqslant\left(\frac{3}{4}-\frac{1}{4} \lambda\right)\left\|\left(L_{2} K^{+}\right) f\right\|^{2} .
\end{gathered}
$$

Remark 1 In Theorem 1, if we take $K=I_{\mathscr{U}}$, and let $\left\{\Lambda_{j}\right\}_{j \in J}$ be a tight $g$-frame for $\mathscr{U}$ with frame bound $A$. Then the canonical dual $\left\{\Gamma_{j}\right\}_{j \in J}$ has the form $\left\{(1 / A) \Lambda_{j}\right\}_{j \in J}$ as $\Gamma_{j}=\Lambda_{j} S^{-1}=(1 / A) \Lambda_{j}$. Then, we can obtain Theorem 2.2 in Ref. 17 from (ii) in Theorem 1 , by taking $\lambda=-1$.

Corollary 1 Let $\left\{\Lambda_{j}\right\}_{j \in J}$ be a g-Bessel sequence in $\mathscr{U}$ w.r.t $\left\{\mathscr{V}_{j}: j \in J\right\}$, with frame operator $S$. If $R(S)$ is closed, we obtain:

(i) For any $f \in R(S),\left\{a_{j}\right\}_{j \in J} \in l^{\infty}(J)$, and $\lambda \in$ $[0,1]$,

$$
\begin{aligned}
& \left\|\sum_{j \in J} a_{j} \Lambda_{j}^{*} \Lambda_{j} S^{+} f\right\|^{2}+2 \lambda \operatorname{Re} \sum_{j \in J}\left(1-a_{j}\right)\left\langle\Lambda_{j} S^{+} f, \Lambda_{j} f\right\rangle \\
& =\left\|\sum_{j \in J}\left(1-a_{j}\right) \Lambda_{j}^{*} \Lambda_{j} S^{+} f\right\|^{2} \\
& \quad+2(1-\lambda) \operatorname{Re} \sum_{j \in J} a_{j}\left\langle\Lambda_{j} S^{+} f, \Lambda_{j} f\right\rangle+(2 \lambda-1)\|f\|^{2} \\
& \geqslant\left(1-(\lambda-1)^{2}\right)\|f\|^{2} .
\end{aligned}
$$

(ii) For any $f \in R(S),\left\{a_{j}\right\}_{j \in J} \in l^{\infty}(J), \lambda \in \mathbb{R}$, and $\lambda \in[-1,3]$,

$$
\begin{aligned}
& \left\|\sum_{j \in J} a_{j} \Lambda_{j}^{*} \Lambda_{j} S^{+} f\right\|^{2}+\lambda \sum_{j \in J} a_{j}\left\langle\Lambda_{j} S^{+} f, \Lambda_{j} f\right\rangle \\
& \quad+\sum_{j \in J}\left(1-\bar{a}_{j}\right)\left\langle\Lambda_{j} f, \Lambda_{j} S^{+} f\right\rangle \\
& =\left\|\sum_{j \in J}\left(1-a_{j}\right) \Lambda_{j}^{*} \Lambda_{j} S^{+} f\right\|^{2} \\
& \quad-(1+\lambda) \sum_{j \in J}\left(1-a_{j}\right)\left\langle\Lambda_{j} S^{+} f, \Lambda_{j} f\right\rangle+(\lambda+1)\|f\|^{2},
\end{aligned}
$$

and

$$
\begin{gathered}
\left\|\sum_{j \in J} a_{j} \Lambda_{j}^{*} \Lambda_{j} S^{+} f\right\|^{2}+\lambda \operatorname{Re} \sum_{j \in J} a_{j}\left\langle\Lambda_{j} S^{+} f, \Lambda_{j} f\right\rangle \\
+\operatorname{Re} \sum_{j \in J}\left(1-\bar{a}_{j}\right)\left\langle\Lambda_{j} f, \Lambda_{j} S^{+} f\right\rangle \\
\geqslant\left(1-\frac{1}{4}(\lambda-1)^{2}\right)\|f\|^{2} \text { or } \\
\left(\frac{3}{4}-\frac{1}{4} \lambda\right)\left\|\sum_{j \in J}\left(1-a_{j}\right) \Lambda_{j}^{*} \Lambda_{j} S^{+} f\right\|^{2} .
\end{gathered}
$$

Proof: From Proposition 1, we know that $\left\{\Lambda_{j}\right\}_{j \in J}$ is an $S$-dual of $\left\{\Lambda_{j}\right\}_{j \in J}$, where $S$ is the frame operator of $\left\{\Lambda_{j}\right\}_{j \in J}$. Hence we can obtain the results from Theorem 1 .

If we take $\lambda=1 / 2$ in (i) or $\lambda=0$ in (ii) in Theorem 1 , we obtain the following corollary.

Corollary 2 Let $\left\{\Lambda_{j}\right\}_{j \in J}$ be a K-g-frame for $\mathscr{U}$ w.r.t $\left\{\mathscr{V}_{j}: j \in J\right\}$. Let $\left\{\Gamma_{j}\right\}_{j \in J}$ be a $g$-Bessel sequence in $\mathscr{U}$ w.r.t. $\left\{\mathscr{V}_{j}: j \in J\right\}$, and be a $K$-dual of $\left\{\Lambda_{j}\right\}_{j \in J}$. If $R(K)$ is closed, then for any $f \in R(K)$ and $\left\{a_{j}\right\}_{j \in J} \in l^{\infty}(J)$, we have

$$
\begin{aligned}
& \sum_{j \in J}\left(1-\bar{a}_{j}\right)\left\langle\Lambda_{j} f, \Gamma_{j} K^{+} f\right\rangle+\left\|\sum_{j \in J} a_{j} \Lambda_{j}^{*} \Gamma_{j} K^{+} f\right\|^{2}= \\
& \sum_{j \in J} a_{j}\left\langle\Gamma_{j} K^{+} f, \Lambda_{j} f\right\rangle+\left\|\sum_{j \in J}\left(1-a_{j}\right) \Lambda_{j}^{*} \Gamma_{j} K^{+} f\right\|^{2},
\end{aligned}
$$

$$
\begin{array}{r}
\operatorname{Re} \sum_{j \in J}\left(1-\bar{a}_{j}\right)\left\langle\Lambda_{j} f, \Gamma_{j} K^{+} f\right\rangle+\left\|\sum_{j \in J} a_{j} \Lambda_{j}^{*} \Gamma_{j} K^{+} f\right\|^{2} \\
\geqslant \frac{3}{4}\|f\|^{2}
\end{array}
$$

$$
\operatorname{Re} \sum_{j \in J}\left(1-a_{j}\right)\left\langle\Gamma_{j} K^{+} f, \Lambda_{j} f\right\rangle+\frac{1}{4}\left\|\sum_{j \in J} a_{j} \Lambda_{j}^{*} \Gamma_{j} K^{+} f\right\|^{2}
$$


Proof: (19) is trivial. If we take $\lambda=0$ in (ii) in Theorem 1, then from (10) and (13), we have for any $f \in R(K)$,

$$
\begin{aligned}
\left\|\sum_{j \in J} a_{j} \Lambda_{j}^{*} \Gamma_{j} K^{+} f\right\|^{2}+\sum_{j \in J}\left(1-\bar{a}_{j}\right)\left\langle\Lambda_{j} f, \Gamma_{j} K^{+} f\right\rangle \\
=\left\|\sum_{j \in J}\left(1-a_{j}\right) \Lambda_{j}^{*} \Gamma_{j} K^{+} f\right\|^{2} \\
\quad-\sum_{j \in J}\left(1-a_{j}\right)\left\langle\Gamma_{j} K^{+} f, \Lambda_{j} f\right\rangle+\|f\|^{2} \\
=\left\|\sum_{j \in J}\left(1-a_{j}\right) \Lambda_{j}^{*} \Gamma_{j} K^{+} f\right\|^{2}-\left\langle L_{2} K^{+} f, f\right\rangle+\|f\|^{2} \\
=\left\|\sum_{j \in J}\left(1-a_{j}\right) \Lambda_{j}^{*} \Gamma_{j} K^{+} f\right\|^{2}-\left\langle\left. L_{2} K^{+}\right|_{R(K)} f, f\right\rangle+\|f\|^{2} \\
=\left\|\sum_{j \in J}\left(1-a_{j}\right) \Lambda_{j}^{*} \Gamma_{j} K^{+} f\right\|^{2}+\left\langle\left(I_{R(K)}-\left.L_{2} K^{+}\right|_{R(K)}\right) f, f\right\rangle \\
=\left\|\sum_{j \in J}\left(1-a_{j}\right) \Lambda_{j}^{*} \Gamma_{j} K^{+} f\right\|^{2}+\left\langle\left. L_{1} K^{+}\right|_{R(K)} f, f\right\rangle \\
=\left\|\sum_{j \in J}\left(1-a_{j}\right) \Lambda_{j}^{*} \Gamma_{j} K^{+} f\right\|^{2}+\sum_{j \in J} a_{j}\left\langle\Gamma_{j} K^{+} f, \Lambda_{j} f\right\rangle .
\end{aligned}
$$

Hence (18) holds. For (20), from (13) we have for any $f \in R(K)$,

$$
\begin{aligned}
\operatorname{Re} \sum_{j \in J} & \left(1-a_{j}\right)\left\langle\Gamma_{j} K^{+} f, \Lambda_{j} f\right\rangle+\left\|\sum_{j \in J} a_{j} \Lambda_{j}^{*} \Gamma_{j} K^{+} f\right\|^{2} \\
& =\operatorname{Re}\left\langle\left. L_{2} K^{+}\right|_{R(K)} f, f\right\rangle+\left\|L_{1} K^{+} f\right\|^{2} \\
& =\left\|L_{1} K^{+} f\right\|^{2}-\operatorname{Re}\left\langle\left. L_{1} K^{+}\right|_{R(K)} f, f\right\rangle+\|f\|^{2} \\
& =\left\|L_{1} K^{+} f\right\|^{2}-\operatorname{Re}\left\langle L_{1} K^{+} f, f\right\rangle+\|f\|^{2} \\
& =\left\|f-\frac{1}{2} L_{1} K^{+} f\right\|^{2}+\frac{3}{4}\left\|L_{1} K^{+} f\right\|^{2} \\
& \geqslant \frac{3}{4}\left\|L_{1} K^{+} f\right\|^{2}=\frac{3}{4}\left\|\sum_{j \in J} a_{j} \Lambda_{j}^{*} \Gamma_{j} K^{+} f\right\|^{2}
\end{aligned}
$$

Hence (20) holds.

Remark 2 In Corollary 2, if we let $K=I_{\mathscr{U}}$, we conclude that $\left\{\Lambda_{j}\right\}_{j \in J}$ is a $g$-frame for $\mathscr{U}$, with an alternate dual $g$-frame $\left\{\Gamma_{j}\right\}_{j \in J}$. Furthermore, if we take $\Lambda_{j} f=w_{i} \pi_{\mathscr{W}_{i}} f, \forall j \in J$, where $\left\{\mathscr{W}_{i}\right\}_{i \in I}$ is a sequence of closed subspaces in $\mathscr{U}$ and $\left\{w_{i}\right\}_{i \in I}$ is a family of positive weights, then it follows that $\left\{\left(\mathscr{W}_{i}, w_{i}\right)\right\}_{i \in I}$ is a fusion frame for $\mathscr{U}$. We now obtain Theorem 4.2 in Ref. 16 from (18) in Corollary 2.

If we take $\left\{a_{j}\right\}_{j \in J}$ in some particular case, then from Theorem 1 we obtain the following result.
Corollary 3 Let $\left\{\Lambda_{j}\right\}_{j \in J}$ be a $K$-g-frame for $\mathscr{U}$ w.r.t $\left\{\mathscr{V}_{j}: j \in J\right\}$. Let $\left\{\Gamma_{j}\right\}_{j \in J}$ be a $g$-Bessel sequence in $\mathscr{U}$ w.r.t. $\left\{\mathscr{V}_{j}: j \in J\right\}$, and be a $K$-dual of $\left\{\Lambda_{j}\right\}_{j \in J}$. If $R(K)$ is closed, then:

(i) For any $f \in R(K), I \subset J$, and $\lambda \in[0,1]$,

$$
\begin{aligned}
\left\|\sum_{j \in I^{\mathrm{c}}} \Lambda_{j}^{*} \Gamma_{j} K^{+} f\right\|^{2}+2 \lambda \operatorname{Re} \sum_{j \in I}\left\langle\Gamma_{j} K^{+} f, \Lambda_{j} f\right\rangle \\
=\left\|\sum_{j \in I} \Lambda_{j}^{*} \Gamma_{j} K^{+} f\right\|^{2}+2(1-\lambda) \operatorname{Re} \sum_{j \in I^{\mathrm{c}}}\left\langle\Gamma_{j} K^{+} f, \Lambda_{j} f\right\rangle \\
\quad+(2 \lambda-1)\|f\|^{2} \\
\geqslant\left(1-(\lambda-1)^{2}\right)\|f\|^{2} .
\end{aligned}
$$

(ii) For any $f \in R(K), \lambda \in \mathbb{R}$, and $\lambda \in[-1,3], I \subset J$,

$$
\begin{aligned}
\left\|\sum_{j \in I^{\mathrm{c}}} \Lambda_{j}^{*} \Gamma_{j} K^{+} f\right\|^{2}+\lambda \sum_{j \in I^{\mathrm{c}}}\left\langle\Gamma_{j} K^{+} f, \Lambda_{j} f\right\rangle+\sum_{j \in I}\left\langle\Lambda_{j} f, \Gamma_{j} K^{+} f\right\rangle \\
=\left\|\sum_{j \in I} \Lambda_{j}^{*} \Gamma_{j} K^{+} f\right\|^{2} \\
\quad-(1+\lambda) \sum_{j \in I}\left\langle\Gamma_{j} K^{+} f, \Lambda_{j} f\right\rangle+(\lambda+1)\|f\|^{2} .
\end{aligned}
$$

Furthermore, we obtain

$$
\begin{aligned}
&\left\|\sum_{j \in I^{c}} \Lambda_{j}^{*} \Gamma_{j} K^{+} f\right\|^{2}+\lambda \operatorname{Re} \sum_{j \in I^{c}}\left\langle\Gamma_{j} K^{+} f, \Lambda_{j} f\right\rangle \\
&+\operatorname{Re} \sum_{j \in I}\left\langle\Lambda_{j} f, \Gamma_{j} K^{+} f\right\rangle \\
& \geqslant\left(1-\frac{1}{4}(\lambda-1)^{2}\right)\|f\|^{2} \operatorname{or}\left(\frac{3}{4}-\frac{1}{4} \lambda\right)\left\|\sum_{j \in I} \Lambda_{j}^{*} \Gamma_{j} K^{+} f\right\|^{2} .
\end{aligned}
$$

Proof: For any subset $I \subset J$, if we take $\left\{a_{j}\right\}_{j \in J}$ in Theorem 1 as

$$
a_{j}= \begin{cases}0, & j \in I, \\ 1, & j \in I^{\mathrm{c}},\end{cases}
$$

then the results follow from Theorem 1 .

Remark 3 In Corollary 3, if we take $K=I_{\mathscr{U}}, \Gamma_{j}=$ $\Lambda_{j}, \forall j \in J$, and $\lambda=1 / 2$ in (i), or $\lambda=-1$ in (ii), then we obtain Theorem 3.2 in Ref. 19 for the case of Parseval $g$-frames. Furthermore, if we take $\lambda=$ $1 / 2$ in (i), or $\lambda=0$ in (ii), we obtain the version of Parseval $g$-frames inequality for Corollary 3.3 in Ref. 19. Also, if we take $K=I_{\mathscr{U}}, \Lambda_{j} f=\left\langle f, f_{j}\right\rangle e_{j}$, $\forall j \in J$, where $\left\{e_{j}\right\}_{j \in J}$ is an orthonormal basis for $\mathscr{U}$, then $\left\{\Lambda_{j}\right\}_{j \in J}$ being an $I_{\mathscr{U}^{\prime}}$-g-frame for $\mathscr{U}$ implies that $\left\{f_{j}\right\}_{j \in J}$ is a frame for $\mathscr{U}$. Now letting $\lambda=1 / 2$ in (i), and $\lambda=-1$ in (ii), we respectively obtain Theorem 3.2 in Ref. 14 and Theorem 2.2 in Ref. 15 from Corollary 3. 
Now we present a parallel result to Theorem 1 in which the elements are restricted to $R\left(K^{+}\right)$.

Theorem 2 Let $\left\{\Lambda_{j}\right\}_{j \in J}$ be a K-g-frame for $\mathscr{U}$ w.r.t $\left\{\mathscr{V}_{j}: j \in J\right\}$. Let $\left\{\Gamma_{j}\right\}_{j \in J}$ be a $g$-Bessel sequence in $\mathscr{U}$ w.r.t. $\left\{\mathscr{V}_{j}: j \in J\right\}$, and be a $K$-dual of $\left\{\Lambda_{j}\right\}_{j \in J}$. If $R(K)$ is closed, then we have:

(i) For $f \in R\left(K^{+}\right),\left\{a_{j}\right\}_{j \in J} \in l^{\infty}(J)$, and $\lambda \in[0,1]$,

$$
\begin{aligned}
& \left\|\sum_{j \in J} a_{j} K^{+} \Lambda_{j}^{*} \Gamma_{j} f\right\|^{2}+2 \lambda \operatorname{Re} \sum_{j \in J}\left(1-a_{j}\right)\left\langle\Gamma_{j} f, \Lambda_{j}\left(K^{+}\right)^{*} f\right\rangle \\
& =\left\|\sum_{j \in J}\left(1-a_{j}\right) K^{+} \Lambda_{j}^{*} \Gamma_{j} f\right\|^{2} \\
& \quad+2(1-\lambda) \operatorname{Re} \sum_{j \in J} a_{j}\left\langle\Gamma_{j} f, \Lambda_{j}\left(K^{+}\right)^{*} f\right\rangle+(2 \lambda-1)\|f\|^{2} \\
& \quad \geqslant\left(1-(\lambda-1)^{2}\right)\|f\|^{2} .
\end{aligned}
$$

(ii) For any $f \in R\left(K^{+}\right),\left\{a_{j}\right\}_{j \in J} \in l^{\infty}(J), \lambda \in \mathbb{R}$, and $\lambda \in[-1,3]$,

$$
\begin{gathered}
\left\|\sum_{j \in J} a_{j} K^{+} \Lambda_{j}^{*} \Gamma_{j} f\right\|^{2}+\lambda \sum_{j \in J} a_{j}\left\langle\Gamma_{j} f, \Lambda_{j}\left(K^{+}\right)^{*} f\right\rangle \\
+\sum_{j \in J}\left(1-\bar{a}_{j}\right)\left\langle\Lambda_{j}\left(K^{+}\right)^{*} f, \Gamma_{j} f\right\rangle \\
=\left\|\sum_{j \in J}\left(1-a_{j}\right) K^{+} \Lambda_{j}^{*} \Gamma_{j} f\right\|^{2} \\
-(1+\lambda) \sum_{j \in J}\left(1-a_{j}\right)\left\langle\Gamma_{j} f, \Lambda_{j}\left(K^{+}\right)^{*} f\right\rangle+(\lambda+1)\|f\|^{2} .
\end{gathered}
$$

Furthermore, we obtain

$$
\begin{gathered}
\left\|\sum_{j \in J} a_{j} K^{+} \Lambda_{j}^{*} \Gamma_{j} f\right\|^{2}+\lambda \operatorname{Re} \sum_{j \in J} a_{j}\left\langle\Gamma_{j} f, \Lambda_{j}\left(K^{+}\right)^{*} f\right\rangle \\
+\operatorname{Re} \sum_{j \in J}\left(1-\bar{a}_{j}\right)\left\langle\Lambda_{j}\left(K^{+}\right)^{*} f, \Gamma_{j} f\right\rangle \\
\geqslant\left(1-\frac{1}{4}(\lambda-1)^{2}\right)\|f\|^{2} \text { or } \\
\left(\frac{3}{4}-\frac{1}{4} \lambda\right)\left\|\sum_{j \in J}\left(1-a_{j}\right) K^{+} \Lambda_{j}^{*} \Gamma_{j} f\right\|^{2} .
\end{gathered}
$$

Proof: Let $L_{1}$ and $L_{2}$ be defined as in Theorem 1. Combining with Lemma 1 and (11), we have

$$
K^{+} L_{1}+K^{+} L_{2}=K^{+} K=P_{R\left(K^{+}\right)} .
$$

It follows that

$$
\left.K^{+} L_{1}\right|_{R\left(K^{+}\right)}+\left.K^{+} L_{2}\right|_{R\left(K^{+}\right)}=I_{R\left(K^{+}\right)} .
$$

Then, by the same method as in Theorem 1 , we can show that (i) and (ii) hold.

From Theorem 2 we also obtain several corollaries as follows.
Corollary 4 Let $\left\{\Lambda_{j}\right\}_{j \in J}$ be a g-Bessel sequence in $\mathscr{U}$ w.r.t $\left\{\mathscr{V}_{j}: j \in J\right\}$ with frame operator $S$. If $R(S)$ is closed, then we have:

(i) For $f \in R\left(S^{+}\right),\left\{a_{j}\right\}_{j \in J} \in l^{\infty}(J)$, and $\lambda \in[0,1]$,

$$
\begin{aligned}
& \left\|\sum_{j \in J} a_{j} S^{+} \Lambda_{j}^{*} \Lambda_{j} f\right\|^{2}+2 \lambda \operatorname{Re} \sum_{j \in J}\left(1-a_{j}\right)\left\langle\Lambda_{j} f, \Lambda_{j}\left(S^{+}\right)^{*} f\right\rangle \\
& =\left\|\sum_{j \in J}\left(1-a_{j}\right) S^{+} \Lambda_{j}^{*} \Lambda_{j} f\right\|^{2} \\
& +2(1-\lambda) \operatorname{Re} \sum_{j \in J} a_{j}\left\langle\Lambda_{j} f, \Lambda_{j}\left(S^{+}\right)^{*} f\right\rangle+(2 \lambda-1)\|f\|^{2} \\
& \geqslant\left(1-(\lambda-1)^{2}\right)\|f\|^{2} .
\end{aligned}
$$

$$
\begin{aligned}
& \left\|\sum_{j \in J} a_{j} S^{+} \Lambda_{j}^{*} \Lambda_{j} f\right\|^{2}+\lambda \sum_{j \in J} a_{j}\left\langle\Lambda_{j} f, \Lambda_{j}\left(S^{+}\right)^{*} f\right\rangle \\
& +\sum_{j \in J}\left(1-\bar{a}_{j}\right)\left\langle\Lambda_{j}\left(S^{+}\right)^{*} f, \Lambda_{j} f\right\rangle \\
& =\left\|\sum_{j \in J}\left(1-a_{j}\right) S^{+} \Lambda_{j}^{*} \Lambda_{j} f\right\|^{2} \\
& -(1+\lambda) \sum_{j \in J}\left(1-a_{j}\right)\left\langle\Lambda_{j} f, \Lambda_{j}\left(S^{+}\right)^{*} f\right\rangle+(\lambda+1)\|f\|^{2}, \\
& \left\|\sum_{j \in J} a_{j} S^{+} \Lambda_{j}^{*} \Lambda_{j} f\right\|^{2}+\lambda \operatorname{Re} \sum_{j \in J} a_{j}\left\langle\Lambda_{j} f, \Lambda_{j}\left(S^{+}\right)^{*} f\right\rangle \\
& +\operatorname{Re} \sum_{j \in J}\left(1-\bar{a}_{j}\right)\left\langle\Lambda_{j}\left(S^{+}\right)^{*} f, \Lambda_{j} f\right\rangle \\
& \geqslant\left(1-\frac{1}{4}(\lambda-1)^{2}\right)\|f\|^{2} \quad \text { or } \\
& \left(\frac{3}{4}-\frac{1}{4} \lambda\right)\left\|\sum_{j \in J}\left(1-a_{j}\right) S^{+} \Lambda_{j}^{*} \Lambda_{j} f\right\|^{2} .
\end{aligned}
$$

Proof: The results follow from Proposition 1 and Theorem 2.

Corollary 5 Let $\left\{\Lambda_{j}\right\}_{j \in J}$ be a K-g-frame for $\mathscr{U}$ w.r.t $\left\{\mathscr{V}_{j}: j \in J\right\}$. Let $\left\{\Gamma_{j}\right\}_{j \in J}$ be a $g$-Bessel sequence in $\mathscr{U}$ w.r.t. $\left\{\mathscr{V}_{j}: j \in J\right\}$, and be a $K$-dual of $\left\{\Lambda_{j}\right\}_{j \in J}$. If $R(K)$ is closed, then for any $f \in R\left(K^{+}\right)$and $\left\{a_{j}\right\}_{j \in J} \in$ $l^{\infty}(J)$, we have

$$
\begin{aligned}
& \sum_{j \in J}\left(1-a_{j}\right)\left\langle\Gamma_{j} f, \Lambda_{j}\left(K^{+}\right)^{*} f\right\rangle+\left\|\sum_{j \in J} a_{j} K^{+} \Lambda_{j}^{*} \Gamma_{j} f\right\|^{2} \\
& =\sum_{j \in J} \bar{a}_{j}\left\langle\Lambda_{j}\left(K^{+}\right)^{*} f, \Gamma_{j} f\right\rangle+\left\|\sum_{j \in J}\left(1-a_{j}\right) K^{+} \Lambda_{j}^{*} \Gamma_{j} f\right\|^{2},
\end{aligned}
$$




$$
\begin{gathered}
\operatorname{Re} \sum_{j \in J}\left(1-a_{j}\right)\left\langle\Gamma_{j} f, \Lambda_{j}\left(K^{+}\right)^{*} f\right\rangle+\left\|\sum_{j \in J} a_{j} K^{+} \Lambda_{j}^{*} \Gamma_{j} f\right\|^{2} \\
\geqslant \frac{3}{4}\|f\|^{2},
\end{gathered}
$$

$$
\operatorname{Re} \sum_{j \in J}\left(1-a_{j}\right)\left\langle\Gamma_{j} f, \Lambda_{j}\left(K^{+}\right)^{*} f\right\rangle+\frac{1}{4}\left\|\sum_{j \in J} a_{j} K^{+} \Lambda_{j}^{*} \Gamma_{j} f\right\|^{2} \geqslant 0 .
$$

Proof: The proof is similar to that of Corollary 2, so we omit it.

Corollary 6 Let $\left\{\Lambda_{j}\right\}_{j \in J}$ be a K-g-frame for $\mathscr{U}$ w.r.t $\left\{\mathscr{V}_{j}: j \in J\right\}$. Let $\left\{\Gamma_{j}\right\}_{j \in J}$ be a $g$-Bessel sequence in $\mathscr{U}$ w.r.t. $\left\{\mathscr{V}_{j}: j \in J\right\}$, and be a $K$-dual of $\left\{\Lambda_{j}\right\}_{j \in J}$. If $R(K)$ is closed, then we have:

(i) For any $f \in R\left(K^{+}\right), I \subset J$, and $\lambda \in[0,1]$,

$$
\begin{aligned}
& \left\|\sum_{j \in I^{c}} K^{+} \Lambda_{j}^{*} \Gamma_{j} f\right\|^{2}+2 \lambda \operatorname{Re} \sum_{j \in I}\left\langle\Gamma_{j} f, \Lambda_{j}\left(K^{+}\right)^{*} f\right\rangle \\
& =\left\|\sum_{j \in I} K^{+} \Lambda_{j}^{*} \Gamma_{j} f\right\|^{2} \\
& +2(1-\lambda) \operatorname{Re} \sum_{j \in I^{c}}\left\langle\Gamma_{j} f, \Lambda_{j}\left(K^{+}\right)^{*} f\right\rangle+(2 \lambda-1)\|f\|^{2} \\
& \quad \geqslant\left(1-(\lambda-1)^{2}\right)\|f\|^{2} . \quad \text { (24) }
\end{aligned}
$$

(ii) For any $f \in R\left(K^{+}\right), \lambda \in \mathbb{R}$, and $\lambda \in[-1,3]$,

$$
\begin{aligned}
\left\|\sum_{j \in I^{c}} K^{+} \Lambda_{j}^{*} \Gamma_{j} f\right\|^{2} & +\lambda \sum_{j \in I^{c}}\left\langle\Gamma_{j} f, \Lambda_{j}\left(K^{+}\right)^{*} f\right\rangle \\
& +\sum_{j \in I}\left\langle\Lambda_{j}\left(K^{+}\right)^{*} f, \Gamma_{j} f\right\rangle \\
= & \left\|\sum_{j \in I} K^{+} \Lambda_{j}^{*} \Gamma_{j} f\right\|^{2} \\
& -(1+\lambda) \sum_{j \in I}\left\langle\Gamma_{j} f, \Lambda_{j}\left(K^{+}\right)^{*} f\right\rangle+(\lambda+1)\|f\|^{2},
\end{aligned}
$$

and

$$
\begin{gathered}
\left\|\sum_{j \in I^{\mathrm{c}}} K^{+} \Lambda_{j}^{*} \Gamma_{j} f\right\|^{2}+\lambda \operatorname{Re} \sum_{j \in I^{\mathrm{c}}}\left\langle\Gamma_{j} f, \Lambda_{j}\left(K^{+}\right)^{*} f\right\rangle \\
\quad+\operatorname{Re} \sum_{j \in I}\left\langle\Lambda_{j}\left(K^{+}\right)^{*} f, \Gamma_{j} f\right\rangle \\
\geqslant\left(1-\frac{1}{4}(\lambda-1)^{2}\right)\|f\|^{2} \text { or }\left(\frac{3}{4}-\frac{1}{4} \lambda\right)\left\|\sum_{j \in I} K^{+} \Lambda_{j}^{*} \Gamma_{j} f\right\|^{2} .
\end{gathered}
$$

Proof: It follows from Theorem 2 if we take $\left\{a_{j}\right\}_{j \in J}$ as defined in (21).

Remark 4 Note that if we take $K=I_{\mathscr{U}}$, then Theorem 1 and Theorem 2 are the same, and according to Remark 1, Remark 2, and Remark 3 we know from Theorem 2 that many known equalities and inequalities in Refs. 14-17, 19 can also be obtained.

Lastly, we provide some new equalities for a $K$ dual pair $\left\{\Lambda_{j}\right\}_{j \in J}$ and $\left\{\Gamma_{j}\right\}_{j \in J}$.

Theorem 3 Let $\left\{\Lambda_{j}\right\}_{j \in J}$ be a K-g-frame for $\mathscr{U}$ w.r.t $\left\{\mathscr{V}_{j}: j \in J\right\}$. Let $\left\{\Gamma_{j}\right\}_{j \in J}$ be a $g$-Bessel sequence in $\mathscr{U}$ w.r.t. $\left\{\mathscr{V}_{j}: j \in J\right\}$, and be a $K$-dual of $\left\{\Lambda_{j}\right\}_{j \in J}$. Then for any $f \in \mathscr{U}$ and $\left\{a_{j}\right\}_{j \in J} \in l^{\infty}(J)$, we obtain

$$
\begin{array}{r}
\left\|\sum_{j \in J}\left(1-a_{j}\right) \Lambda_{j}^{*} \Gamma_{j} f\right\|^{2}+2 \operatorname{Re} \sum_{j \in J} a_{j}\left\langle\Gamma_{j} f, \Lambda_{j} K f\right\rangle \\
=\left\|\sum_{j \in J} a_{j} \Lambda_{j}^{*} \Gamma_{j} f\right\|^{2}+\|K f\|^{2} .
\end{array}
$$

Proof: Let $L_{1}$ and $L_{2}$ be defined as in Theorem 1. As $\left\{\Gamma_{j}\right\}_{j \in J}$ is a $K$-dual of $\left\{\Lambda_{j}\right\}_{j \in J}$, (11) holds. Regarding $L_{1}$ and $L_{2}$ as $P$ and $Q$, respectively, in Lemma 4, for any $f \in \mathscr{U}$, now we have

$$
\begin{aligned}
\left\langle L_{1}^{*} L_{1} f, f\right\rangle+ & \left\langle K^{*} K f, f\right\rangle \\
& =\left\langle L_{2}^{*} L_{2} f, f\right\rangle+\left\langle K^{*} L_{1} f, f\right\rangle+\left\langle L_{1}^{*} K f, f\right\rangle,
\end{aligned}
$$

that is

$$
\left\|L_{1} f\right\|^{2}+\|K f\|^{2}=\left\|L_{2} f\right\|^{2}+\left\langle L_{1} f, K f\right\rangle+\left\langle K f, L_{1} f\right\rangle .
$$

Then we have

$$
\begin{aligned}
&\left\|\sum_{j \in J} a_{j} \Lambda_{j}^{*} \Gamma_{j} f\right\|^{2}+\|K f\|^{2} \\
&=\left\|\sum_{j \in J}\left(1-a_{j}\right) \Lambda_{j}^{*} \Gamma_{j} f\right\|^{2}+\sum_{j \in J} a_{j}\left\langle\Gamma_{j} f, \Lambda_{j} K f\right\rangle \\
&+\sum_{j \in J} \bar{a}_{j}\left\langle\Lambda_{j} K f, \Gamma_{j} f\right\rangle .
\end{aligned}
$$

Now, (25) follows from (26).

From different aspects of Theorem 3, we can obtain the following four corollaries.

Corollary 7 Let $\left\{\Lambda_{j}\right\}_{j \in J}$ be a g-Bessel sequence in $\mathscr{U}$ w.r.t $\left\{\mathscr{V}_{j}: j \in J\right\}$, with frame operator $S$. Then for any $f \in \mathscr{U}$ and $\left\{a_{j}\right\}_{j \in J} \in l^{\infty}(J)$, we obtain

$$
\begin{aligned}
\left\|\sum_{j \in J}\left(1-a_{j}\right) \Lambda_{j}^{*} \Lambda_{j} f\right\|^{2}+ & 2 \operatorname{Re} \sum_{j \in J} a_{j}\left\langle\Lambda_{j} f, \Lambda_{j} S f\right\rangle \\
& =\left\|\sum_{j \in J} a_{j} \Lambda_{j}^{*} \Lambda_{j} f\right\|^{2}+\|S f\|^{2} .
\end{aligned}
$$

Proof: For any $g$-Bessel sequence $\left\{\Lambda_{j}\right\}_{j \in J}$ with frame operator $S,\left\{\Lambda_{j}\right\}_{j \in J}$ is an $S$-dual of $\left\{\Lambda_{j}\right\}_{j \in J}$. Hence the result follows from Theorem 3 . 
Corollary 8 Let $\left\{\Lambda_{j}\right\}_{j \in J}$ be a K-g-frame for $\mathscr{U}$ w.r.t $\left\{\mathscr{V}_{j}: j \in J\right\}$. Let $\left\{\Gamma_{j}\right\}_{j \in J}$ be a $g$-Bessel sequence in $\mathscr{U}$ w.r.t. $\left\{\mathscr{V}_{j}: j \in J\right\}$, and be a K-dual of $\left\{\Lambda_{j}\right\}_{j \in J}$. Then for any $f \in \mathscr{U}$ and any subset $I \subset J$, we obtain

$$
\begin{aligned}
\left\|\sum_{j \in I} \Lambda_{j}^{*} \Gamma_{j} f\right\|^{2}+2 \operatorname{Re} \sum_{j \in I^{\mathrm{c}}}\left\langle\Gamma_{j} f, \Lambda_{j} K f\right\rangle & \\
& =\left\|\sum_{j \in I^{\mathrm{c}}} \Lambda_{j}^{*} \Gamma_{j} f\right\|^{2}+\|K f\|^{2} .
\end{aligned}
$$

Proof: The result follows by taking $\left\{a_{j}\right\}_{j \in J}$ as in (21).

Corollary 9 Let $\left\{\Lambda_{j}\right\}_{j \in J}$ be a K-g-frame for $\mathscr{U}$ w.r.t $\left\{\mathscr{V}_{j}: j \in J\right\}$. Let $\left\{\Gamma_{j}\right\}_{j \in J}$ be a $g$-Bessel sequence in $\mathscr{U}$ w.r.t. $\left\{\mathscr{V}_{j}: j \in J\right\}$, and be a $K$-dual of $\left\{\Lambda_{j}\right\}_{j \in J}$. If $R(K)$ is closed, then for any $f \in R(K)$ and $\left\{a_{j}\right\}_{j \in J} \in l^{\infty}(J)$, we obtain

$$
\begin{aligned}
\left\|\sum_{j \in J}\left(1-a_{j}\right) \Lambda_{j}^{*} \Gamma_{j} K^{+} f\right\|^{2} & +2 \operatorname{Re} \sum_{j \in J} a_{j}\left\langle\Gamma_{j} K^{+} f, \Lambda_{j} f\right\rangle \\
& =\left\|\sum_{j \in J} a_{j} \Lambda_{j}^{*} \Gamma_{j} K^{+} f\right\|^{2}+\|f\|^{2} .
\end{aligned}
$$

Proof: The proof is similar to that of Theorem 1, so we omit it.

Corollary 10 Let $\left\{\Lambda_{j}\right\}_{j \in J}$ be a $K$-g-frame for $\mathscr{U}$ w.r.t $\left\{\mathscr{V}_{j}: j \in J\right\}$. Let $\left\{\Gamma_{j}\right\}_{j \in J}$ be a $g$-Bessel sequence in $\mathscr{U}$ w.r.t. $\left\{\mathscr{V}_{j}: j \in J\right\}$, and be a $K$-dual of $\left\{\Lambda_{j}\right\}_{j \in J}$. If $R(K)$ is closed, then for any $f \in R\left(K^{+}\right)$and $\left\{a_{j}\right\}_{j \in J} \in$ $l^{\infty}(\mathrm{J})$, we obtain

$$
\begin{aligned}
\left\|\sum_{j \in J}\left(1-a_{j}\right) K^{+} \Lambda_{j}^{*} \Gamma_{j} f\right\|^{2} & +2 \operatorname{Re} \sum_{j \in J} a_{j}\left\langle\Gamma_{j} f, \Lambda_{j}\left(K^{+}\right)^{*} f\right\rangle \\
& =\left\|\sum_{j \in J} a_{j} K^{+} \Lambda_{j}^{*} \Gamma_{j} f\right\|^{2}+\|f\|^{2} .
\end{aligned}
$$

Proof: The proof is similar to that of Theorem 2, so we omit it.

Acknowledgements: This work is supported by the projects of Xiamen University of Technology (Grant Nos. G2017005 and 50419004).

\section{REFERENCES}

1. Sun W (2006) G-frames and g-Riesz bases. J Math Anal Appl 322, 437-452.

2. Christensen O (2003) An introduction to Frames and Riesz bases. Birkhäuser, Boston.

3. Li S, Ogawa H (2004) Pseudoframes for subspaces with applications. J Fourier Anal Appl 10, 409-431.

4. Casazza PG, Kutyniok G (2004) Frames of subspaces. In: Heil C, Jorgensen PET, Larson DR (eds) Wavelets, Frames and Operator Theory, Contemp Math 345, AMS, Rhode Island, pp 87-113.

5. Casazza PG, Kutyniok G, Li S (2008) Fusion frames and distributed processing. Appl Comput Harmon Anal 25, 114-132.

6. Khosravi A, Musazadeh K (2008) Fusion frames and g-frames. J Math Anal Appl 342, 1068-1083.

7. Li JZ, Zhu YC (2011) Exact g-frames in Hilbert spaces. J Math Anal Appl 374, 201-209.

8. Yu BY, Shu ZB (2011) Construction of dual g-frames for closed subspaces. Int $J$ Wavelets Multiresolut Inf Process 9, 947-964.

9. Wang YJ, Zhu YC (2009) G-frames and g-frame sequences in Hilbert spaces. Acta Math Sin Engl Ser 25, 2093-2106.

10. Xiao XC, Zhu YC (2017) Exact $K$-g-Frames in Hilbert spaces. Results Math 72, 1329-1339.

11. Zhu YC (2008) Characterizations of g-frames and g-Riesz bases in Hilbert spaces. Acta Math Sin Engl Ser 24, 1727-1736.

12. Xiao XC, Zhu YC, Shu ZB, et al (2015) G-frames with bounded linear operators. Rocky Mountain $J$ Math 45, 675-693.

13. Găvruţa L (2012) Frames for operators. Appl Comp Harm Anal 32, 139-144.

14. Găvruţa P (2006) On some identities and inequalities for frames in Hilbert spaces. $J$ Math Anal Appl 321, 469-478.

15. Zhu XG, Wu GC (2010) A note on some equalities for frames in Hilbert spaces. Appl Math Lett 23, 788-790.

16. Xiao XC, Zhu YC, Ding ML (2015) Erasures and equalities for fusion frames in Hilbert spaces. Bull Malays Math Sci Soc 38, 1035-1045.

17. Li DF, Sun WC (2008) Some equalities and inequalities for generalized frames. Chinese $J$ Contemp Math 29A, 513-518.

18. Xiang ZQ (2016) New inequlites for g-frames in Hilbert $C^{*}$-modules. $J$ Math Inequal 10, 889-897.

19. Poria A (2017) Some identities and inequalities for Hilbert-Schmidt frame. Mediterr J Math 14, ID 59, $1-14$. 